\title{
Suitability of Digital Elevation Models for Watershed Segmenting Images with Directional Illumination
}

\author{
Tahir Q. Syed ${ }^{1}$, V. Vigneron ${ }^{2}$, C. Montagne ${ }^{2}$, S. Lelandais-Bonadè ${ }^{2}$ \\ ${ }^{1}$ National University of Computer and Emerging Sciences, Pakistan \\ ${ }^{2}$ Université d'Evry Val d'Essonne, France
}

\begin{abstract}
This paper investigates the use of different functions for the digital elevation model input to the watershed transform. The use of gradient information is the most frequent one, but its strength varies due to illumination variations. We investigate the two major classes of input functions, distance maps and the gradient, their combinations, and propose an different function using soft clustering memberships that is not covariant with illumination.
\end{abstract}

\section{Keywords:}

watershed transform, digital elevation model, partial class memberships, fuzzy c-means, directional illumination, confocal microscopy

\section{INTRODUCTION}

The watershed algorithm $(W T)$ [9], which has proved very useful in many areas of image segmentation and analysis, is a morphology-based segmentation method [15]. It understands a greyscale image as a topographic surface [3], with valleys and crests corresponding respectively to low and high intensity parts of the image. In this paper we call this representation the topographic image function. If we flood a topographic image from its regional minima, preventing the merge of the waters coming from different sources, the topographic image is eventually separated as two different sets: the catchment basins, and the watershed lines.

Before applying the watershed transform, one must be sure that objects and their background both contain a regional minimum, and that crest lines outline the objects. If not, the original image must be transformed so that the contours to be calculated correspond to watershed lines, and the objects to catchment basins surrounded by them.

The watershed transform is formally defined in terms of skeleton by influence zones [3]. The watershed line is the set of points at equal distance of the image minima, according to a certain distance: the topographic distance. Distance-based formulations of watershed transform are due to Meyer in the discrete case [14] and to Schmidt and Najman in the continuous case [16]. However, the watershed transform is applicable to various transformations of the grey level image information. The usual practice has been to use the gadient of the initial grey valued image as the topographic image [3, 22], since the gradient encodes the structure of the image.
In standard image segmentation applications, contours correspond to high luminance transitions, i.e. points where the gradient norm $\|\nabla f\|$ takes high values. But, when other criteria are relevant, other functions can be used. In particular, when the segmentation is based on the shape of the objects, the distance function is very helpful.

The two image transformations, the distance transform and the gradient transform, have been widely studied and used in different applications of watershed segmentation [12]. In this paper we study the difference between the segmentations produced by both image transforms, and present another image transform that respect local image structure better than the gradient transform by preserving the strength of the contrast where the gradient function decays. This study is important because the segmentation over any topographic function not only defines which regions that are assigned to objects in the image, but also defines the shape and extent of those regions, and sometimes dictates the post-segmentation corrective process as well [1, 8]. Chen et al. and Kaur et al. have produced a similar study while limiting themselves to the various versions of the distance and the gradient transforms alone respectively.

For this work we have chosen a difficult dataset to evidence the problems with the various topographic functions from the perspective of a practical segmentation problem. It has been described in Vigneron et al. [20] and consists of images of cancerous cells cultured in vitro that are non-pigmented and possess similar grey values to the substrate. The images have been exposed to uneven illumination, are noisy an exhibit significant overlap among the cells. In that paper we had proposed several corrective transforms for the data which managed to rectify all problems except for locally directed illumination, and suggested a method to reliably detect the count and locations of the cells, marking them with pseudocentroids which we called cell centres or simply centres. Now we look at how these images could be used to separate the cells using the $W T$ and at more transforms of the image data to make them more amenable to the use of the $W T$.

\section{IMAGE TRANSFORMS FOR THE TOPOGRAPHICAL FUNCTION}

\subsection{The distance transform}

The notion of distance is intrinsic to the watershed transform as the influence zones of regional minima are defined as the geodesic distances between them. The geodesic zone of influence $z_{X}\left(Y_{i}\right)$ of the regional minimum $Y_{i}$ is the set of points $X$ of the image at a fi- 
nite geodesic distance from $Y$ and closer to $Y_{i}$ than to any other $Y_{j}$.

$$
\begin{array}{r}
z_{X}(Y)=x \in X: d_{X}\left(X, Y_{i}\right) \text { finite and } \\
\forall j \neq i, d_{X}\left(X, Y_{i}\right) \leq d_{X}\left(X, Y_{j}\right)
\end{array}
$$

Watershed segmentation applied to distance transformed $(D T)$ binary images (usually binarised through thresholding) is useful for separating touching objects that are convex (see [12, 5, 23]). The points of origin $P$ from which these distances will be calculated could be centroids or other kinds of seeds within objects. The measure of distance is therefore

$$
D T(P)[X]=\min _{Y \in P} \operatorname{dist}(\mathrm{X}, \mathrm{Y})
$$

for each point $x$ in the image to the nearest point $y$ in $P$. The calculation of chamfer distances on binary images is performed in practice by propagating local distances as min-sums forward and backward passes as in the popular SLT algorithm by Rosenfeld and Pfaltz [19]. The distance used is the Eucledian distance i.e. $\operatorname{dist}(X, Y)=\sqrt{\left(x_{1}-y_{1}\right)^{2}+\left(x_{2}-y_{2}\right)^{2}}$ for the $2 D$ dimensions 1,2 .

We notice that watershed segmentation based on $D T$ does not necessarily segment objects right at their boundaries, instead decomposes them in an equitable manner based on the chamfer distance, even though we introduced a prior information in the form of extended $D T$ seeds.

\subsection{The gradient-weighted distance transform}

The gradient transformation is intensity-based and assumes that inter-cellular gradients are higher than intra-cellular ones gradients. As with all gradient-based operations, this transformation is sensitive to imaging noise, and usually results in over-segmentation.

To overcome the above difficulties, namely non-realistic segmentation of $D T$ and the extreme over-segmentation of $G T$, we use a combined image transformation called the "gradient-weighted distance transform" or $G W D T$, which accounts for both geometric and intensity features. The distance element smooths out the many local minima generated by the gradient, by augmenting it with a monotonically-increasing distance component, and the gradient component adds critical boundary information for better placement of watershed boundaries.

The geometric distance transform $D$ and the gradient transform $G$ are combined into a single representation that captures the object separation cues available in the data. One challenge in this regard is the fact that these quantities are dissimilar, but the following formula [11] gives the result of the combining operation in distance units:

$$
D_{w}=D \times \exp \left(1-\frac{G-G_{\min }}{G_{\max }-G_{\min }}\right)
$$

where $G_{\min }$ and $G_{\max }$ are the minimum and maximum values of the gradient $G$ (the Deriche gradient [6] is used) needed for normalization. Note that the distance value $D_{w}$ is high at positions closer to the center of foreground objects, and in pixels with smaller gradient values. $D_{w}$ is smaller close to the boundary of the foreground objects, or where the gradient is relatively large. Intuitively, this captures the essential object separation cue that pixels with bigger gradient values tend to be on the boundary of an isolated object, or on the boundary between two touching objects.

\subsection{Building cell shape priors into the distance map}

Usually only a $D T$ seed's presence is used, the seed's structure remains unemployed. This section presents a method to inprove the outcome of the $D T$ by using the information related to the seeds' placement, shape and size. Such a priori information is commonly exploited in other segmentation schemes such as active contours [10]. Point-seeds would work well if all cells have the same size and shape or if the image function has clearly demarcated object boundaries. But since that is rarely the case, point-markers on a distance map could bias watershed segmentation results for touching objects e.g. cells lying in clusters. We notice that the elevation around peaks of circular cells are more pointy as well as circular, while those of spread cells they form particular longitudinallyconvex crests. Thus the use of regional maxima around centres as watershed markers will allow shape information to be propagated over the distance map. As a seed is always contained within object boundaries, we use a windowed h-dome around each peak to determinse the value of $h$ dynamicallyusing an Otsu [17] threshold. Fig. 1 p) shows an example of the markers thus produced. They compare favorably with the parent cell in Fig. 1 1

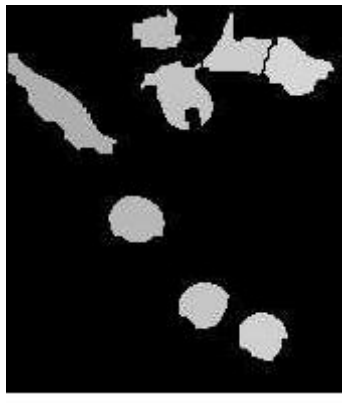

(a)

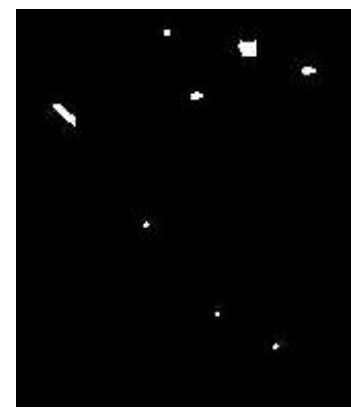

(b)
Fig. 1. Shape relationship between (a) cells and (b) corresponding watershed markers.

The problems however that exist with these methods include:

- the frequent aberrant cases of cells where the gradient information is not strong enough to stop the flooding process, and the watershed thus produced reflects simply the distance map, cutting cells in arbitrary shapes.

-malplaced centres: cell centres detected multiple times on the same cell force the cell to be separated into more than one segments even if left naturally without markers the cell would have formed a unique basin.

- the $D T$ seed might be mal-formed, making the segmentation incoherent with the ground-truth

The following section discusses our method of forming the topographical image that uses neither the gradient nor the distance transform, but instead a partial class assignment of image pixels to create relatively homogeneous regions.

\section{PARTIAL MEMBERSHIP CLUSTERING AS THE TOPOGRAPHIC FUNCTION}

In spite of a slew of modifications available for the watershed transform, little interest has been shown to using a pre-clustered topo- 
graphic image. The authors who have studied this possibility are [7][?], where pixels are represented by the memberships to each class of interest, and subsequently a morphological gradient is computed from this representation on which the $W T$ is applied; and Levner et al. [?], who trained two Bayes classifiers to identify markers and object silhouettes and then combined the results into a probability mapp. However, both methods are supervised using pixel labelings done by experts. The one we present in this section obviates the need for supervision.

Let us recall that one of the main problems in image segmentation is uncertainty, from additive noise, imprecision in computations and vagueness in class definitions. Traditionally, probability theory was the primary mathematical model used to deal with uncertainty problems; however, the possibility concept introduced by the fuzzy set theory has gained popularity in modeling and propagating uncertainty in imaging applications.

Hard clustering divides data into distinct clusters, where each data element belongs to exactly one cluster. Fuzzy (or soft) clustering, data elements can belong to more than one cluster, and associated with each element is a set of membership levels. These indicate the strength of the association between that data element and a particular cluster. Fuzzy clustering is a process of assigning these membership levels, and then using them to assign data elements to one or more clusters. One of the most widely used fuzzy clustering algorithms is the Fuzzy C-Means (FCM) Algorithm [4]. The FCM algorithm attempts to partition a finite collection of $n$ elements $X=x_{1}, \ldots, x_{n}$ into a collection of $c$ fuzzy clusters with respect to some given criterion. Given a finite set of data, the algorithm returns a list of $c$ cluster centres $C=c_{1}, \ldots, c_{c}$ and a partition matrix $U=u_{i, j} \in[0,1], i=1, \ldots, n, j=1, \ldots, c$, where each element $u_{i j}$ tells the degree to which element $x_{i}$ belongs to cluster $c_{j}$. Thus, points on the edge of a cluster, may be in the cluster to a lesser degree than points in the center of cluster. For each point $x$ we have a coefficient giving the degree of being in the $k$ th cluster $u_{k}(x)$. Usually, the sum of those coefficients for any given $x$ is defined to be 1:

$$
\forall x\left(\sum_{k=1}^{\mathrm{c}} u_{k}(x)=1\right) .
$$

With fuzzy $c$-means, the centroid of a cluster is the mean of all points, weighted by their degree of belonging to the cluster:

$$
\operatorname{center}_{k}=\frac{\sum_{x} u_{k}(x) x}{\sum_{x} u_{k}(x)} .
$$

The degree of belonging is related to the inverse of the distance to the cluster center:

$$
u_{k}(x)=\frac{1}{d\left(\operatorname{center}_{k}, x\right)},
$$

The fuzzy $c$-means algorithm is very similar to the $k$-means algorithm:

(1) Choose a number of clusters.

(2) Assign randomly to each point coefficients for being in the clusters.

(3) Repeat until the algorithm has converged (that is, the coefficients' change between two iterations is no more than a sensitivity threshold $\varepsilon$ ): (a) Compute the centroid for each cluster, using the formula above.

(b) For each point, compute its coefficients of being in the clusters, using the formula above.

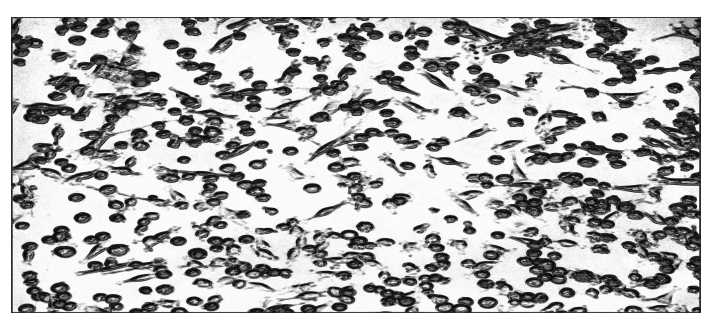

(a)

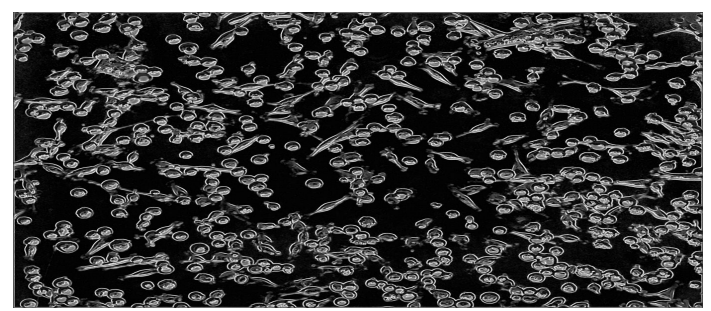

(b)

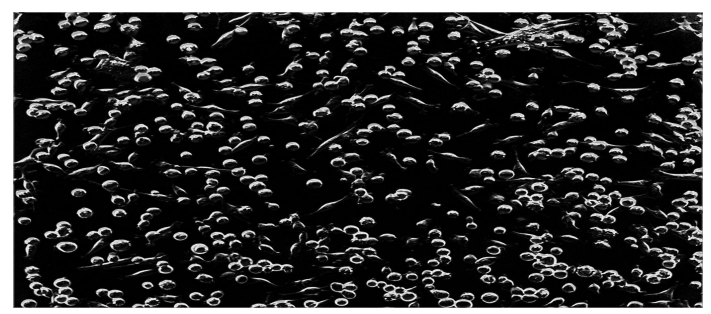

(c)

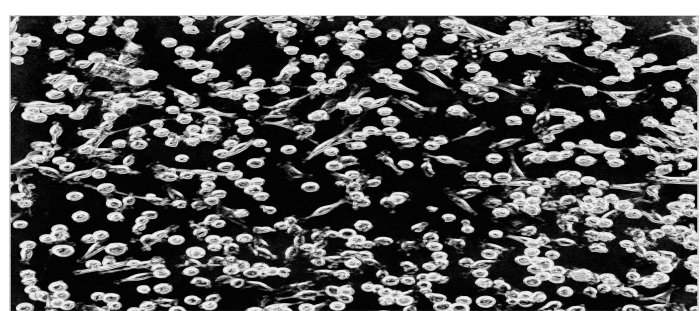

(d)

Fig. 2. Fuzzy- $C$-means class assignment to (a) background (b) cell matter and (c) highly-contrasted cell matter or walls. (d) represents the sum of the scores in (b) and (c) i.e. the net probability of a pixel belonging to the class cell.

Using a very similar idea, we assign one of the three membershipclasses to each pixel in the image: background, cell-inside and cellborders. cell-borders is somewhat of a misnomer because its higher membership degrees represent highly-textured parts of the cells, albeit mostly those near and around its cell walls. However, the manner these degrees are used (post-classification re-combination) renders the difference insignificant. Combined, cell-inside and cellborders represent the cell pixels in the image. The sum of the labels in cell-inside and cell-borders represents our topographic image, 
for convenience henceforth called the probability map. The desirable property of this image is that all regional minima among the cell pixels are higher than their counterparts in the background (yet we still do not have a connected component vs background decision). This means that any method of over-segmentation correction will unify cell regions and background regions more successfully.

\section{ASSESSING THE SUITABILITY OF THE DIFFERENT TOPOGRAPHIC FUNCTIONS THROUGH THE QUALITY OF THE RESULTING SEGMENTATION}

It is necessary to have a way to perform discriminatory assessment of the quality of segmentation produced byeach topologica image function. In this section we present some existing ideas and a natural adaptation of one of them to our situation.

\subsection{Methods of segmentation quality evaluation}

Because of the profusion of image segmentation methods evaluation becomes crucial, but the problem of defining a good segmentation remains unsolved and the solution mainly depends on the goal. A good segmentation can be defined as a segmentation true to one given by a human being, and in the case of real images it must generally be built "by hand" [18] by an expert of the application domain.to extract is not known a priori, there is generally no unique solution to the division of an image into "relevant" regions. The "relevance" of a region is indeed a notion highly dependent on what Correia and Pereira in the same article call an "application scenario". Since for our purposes such a ground-reality is existent we shall limit ourselves to the first class of methods.

Many criteria of discrepancy have been proposed, which can be used when a ground truth is available, usually given by an expert of the application domain, who is supposed to exactly know what he is expecting, in terms of accuracy, level of detail, etc. Among these discrepancy criteria we could cite Vinet measure [21], the measure of Yasnoff et al. [25] which counts the number of mis-segmented pixels, the Baddeley distance [24], and the ultimate measurement accuracy of Zhang [26] and the measure of consistency between segmentations of Martin [13].

\subsection{The discrepancy criterion}

We define a discrepancy criterion where as usual the ground-truth is obtained by delimiting cell boundaries by hand, and the resulting contour and the enclosed region are used as references, using which different segmentations methods can be compared. What does not change is the nature of the segmentation method, i.e. a watershed mosaic of regions. This has the implication that all pixels belonging to the image are accounted for, by falling either within one region or catchment basin or into the background. The ground-truth seves to establish whether the pixel is in the correct basin or is elsewhere. Thus a group of pixels that should have been, according to the ground-truth, in a particular basin, may either be attributed by a segmentation to another, or just as well the other way round. This gives rise to two types of errors in segmentation that the evaluation tries to quantify: basin overflow $(B O)$ beyond cell boundaries, resulting in excessive pixels attributed to a cell; and basin shortfall $(B S)$, i.e. loss of cell pixels to the background or neighbouring basins. Basin overflow is calculated as the ratio of segmented region pixels lying outside of the manual contour, and basin shortfall is calculated as the ratio of the manually cut region pixels not in the segmented region. Thus, if we let $p$ be a pixel and $R_{i}$ a segmented region and $\mathrm{R}_{i}$ its manual equivalent, then the following criteria can be defined:

$$
\begin{array}{r}
\text { Correct Attribution (CA) : } p \in R_{i} \text { and } p \in \mathrm{R}_{i} \\
\text { Basin Overflow (BO) : } p \in R_{i} \text { but } p \notin \mathrm{R}_{i} \\
\text { Basin Shortfall (BS) :p } \in \mathrm{R}_{i} \text { but } p \notin R_{i}
\end{array}
$$

This simple yet handy discrepancy criterion is adequate in our application scenario, as we shall see in the following discussion, since it produces fairly discriminating results.

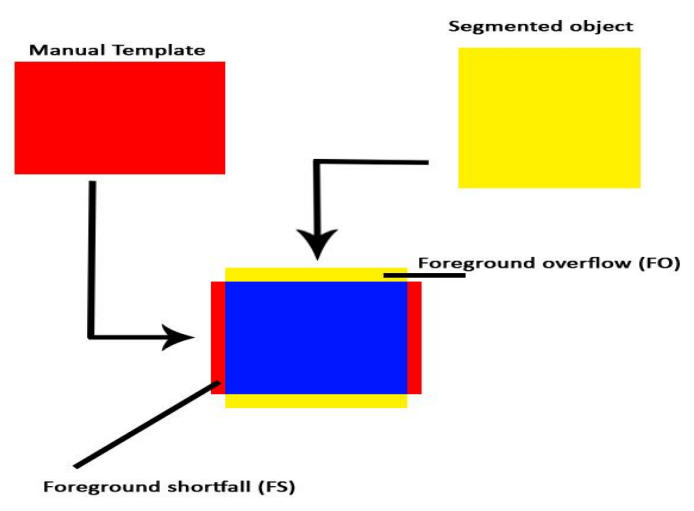

Fig. 3. Illustration of the comparison.

\subsection{Evaluation Methodology}

The test data comprises 42 connected component images that we call subimages, each containing more than one cells in an aggregate. Each has been extracted from our test set of 14 images, i.e. 3 subimages from each image, and therefore represent well the variability in the image data.

The ground-truth (refer to Section 4.2) is established by tracing out the contours of each cell in an subimages by hand. We start with a lasso that is unattached to any point in an image and anchor it to every judged salient point on the cell boundary. No interpolation is performed between any pair of these points and the lasso is rigid i.e. we end up with a piecewise-linear curve.

\subsection{Comparison and discussion}

This part of the section will aim to benchmark the five functions mentioned on the same test data sample so that for the latter part of the work could be carried out on the one that is best suitable for our purposes.

The resulting data thus compiled are too copious to reproduce raw. instead we have graphed them trends in them could be easily spotted. Figures 4 through 5 illustrate the comparisons.

Fig. 4 compares (a) correct attribution $(C A)$, (b) basin overflow $(B O)$ and (c) basin shortfall $(B S)$ for the subimage set. Several trends is noticeable, and it is only fair to discuss them individually and at proper length, as in the following:

- The subimages present a diverse test data set, and we thus obtain non-flat curves with singular corners. 


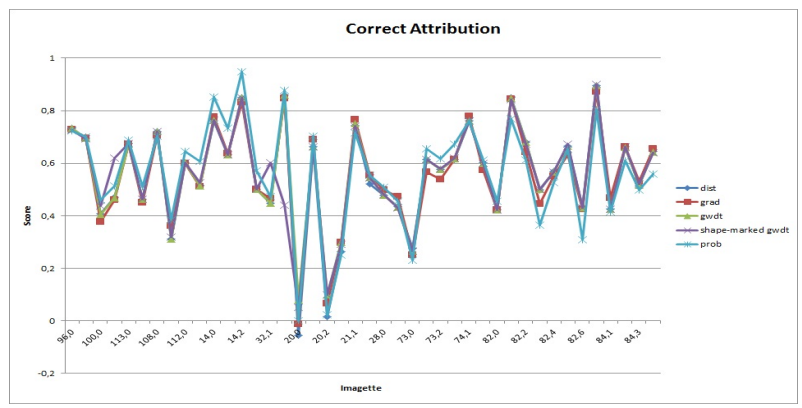

(a)

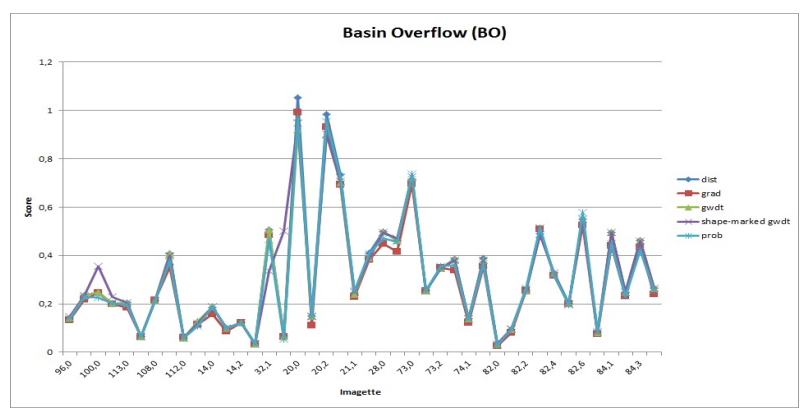

(b)

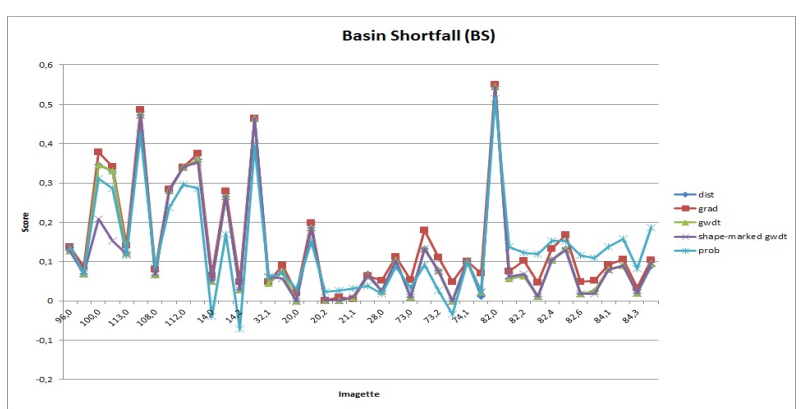

(c)

Fig. 4. Graphs of (a) Correct segmentation (b) basin overflow and (c) basin shortfall for the entire subimage data.

-All curves follow very similar trajectories, since all data lie in tight neighborhoods. The advantage of one topographical function over the others, except for the probability map, is therefore marginal.

- Simple $D T$ does not fare too badly considering its relative lack complexity of calculation when compared to the shape-guided $G W D T$. The first two and the fourth curves practically overlay each other.

- The gradient plays the maverick in (a) and (b), due to its different $B S$. This could be attributable to the noisy nature of the gradient image, which causes the basins to form inside of the highgradient band representing cell walls, thus causing the high $B S$ values and lowering those of $C A$. But at the same time the gradient also demonstrates lower $B O$, which is advantageous for a segmentation in which deeper basins risk spilling over into shallower ones, as can happen in case of the simple chamfer topographic function.
- The probability map demonstrates the highest $C A$ for the majority of subimages, and the lowest $B S$ for the majority of the subimages. This could be attributed to the within-cell and nearcell-wall probabilities being very distinct from the background probabilities.

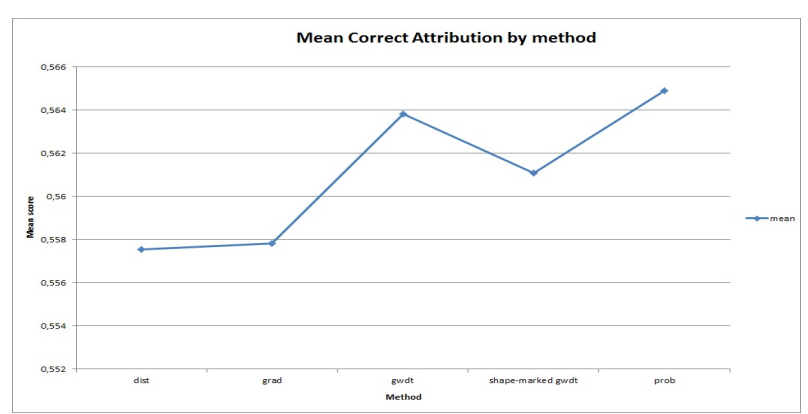

(a)

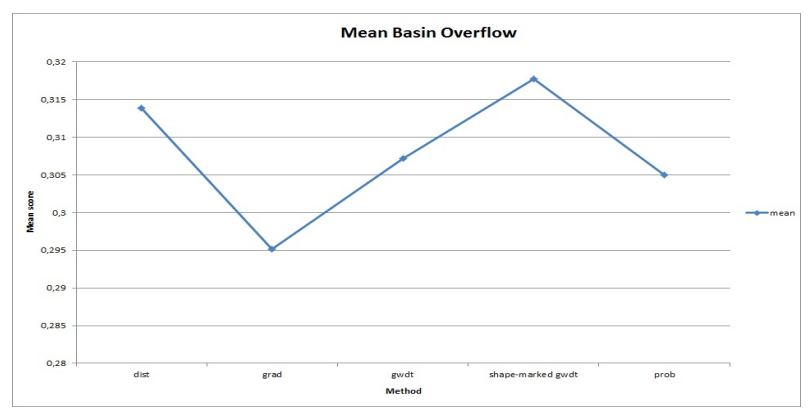

(b)

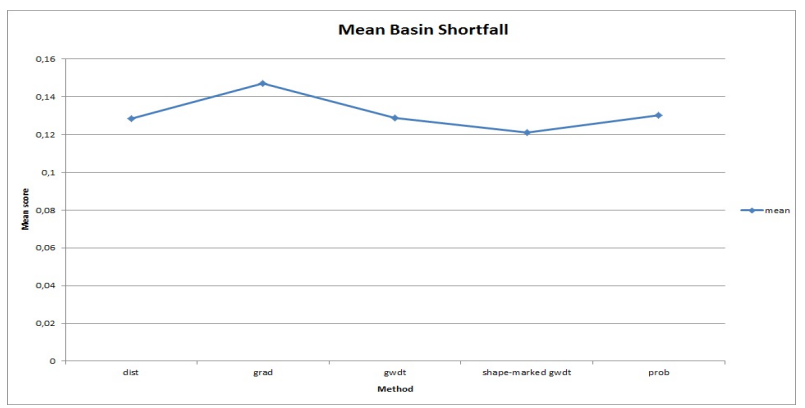

(c)

Fig. 5. Trends in mean of (a) Correct segmentation (b) basin overflow and (c) basin shortfall.

Fig. 5illustrates the trend of the mean values of the segmentation quality parameter scores, in the same order as the previous image:

- The gradient scores the poorest on $C A$ but the best in terms of $B O$. Barring the gradient from the list, we would have flat $C A$ and $B S$ curves hovering around $0.65 \%$ and $0.07 \%$ respectively.

-Probability map shows a slightly superior $C A$ over gradient, but also reasonably superior $B O$ and has a $B S$ that is only slightly worse off compared to all other topographic functions involving a distance measure. 


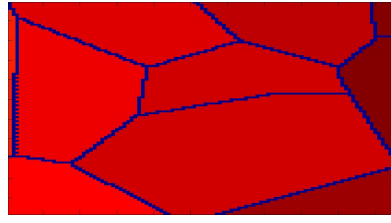

(b)

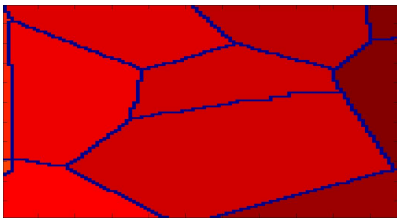

(d)

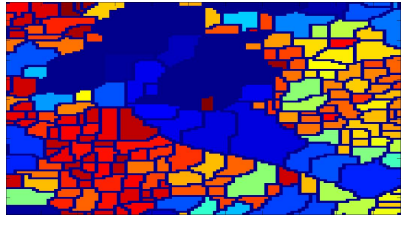

(c)

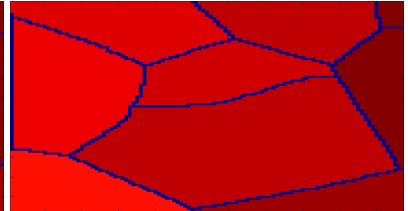

(e)

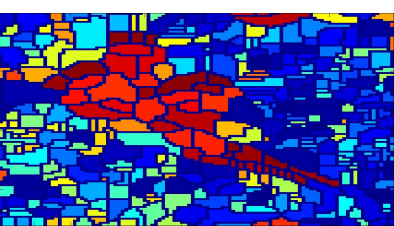

(f)

Fig. 6. Segmentation of the subimage a) 0032.1, using for topographic relief function: b) chamfer distance map, c) Deriche gradient, d) GWDT, e) shape-guided GWDT, and f) fuzzy $C$-means probability map.

Let us revisit the comparison with a visual inspection in Fig. 6 The gradient map (Fig. 6 c.) produces shallower basins than the probability map (Fig. 6 e.), and these being less distinguished from those in the background and are therefore difficult to correctly unify into cell objects through the use of corrective post-segmentationprocessing as would be required after this work. Moreover, the former exceed the cell contours and into the background, while the latter do not. This reinforces the decision arrived using the discrepancy criterion to use the probability map as the topographic function.

\section{CONCLUSIONS AND PERSPECTIVE}

This paper compares various topographic functions that could be uses in order to achieve desirable image segmetation using the watershed transform, and at the same time shows how ill-adapted some of them are for applying to image data from a real-world application which have been acquired in a relaxed-control envirenment. It then presents a new topographic function using partial class assignment of image pixels into object parts and the background. Our function produces superior results to both the distance and the gradient transforms. It repects image structure like the grandient transform but remains unaffected by illumination variations that disturbs gradient information aorund lit and sombre object countours. It does not however alleviate the proble of over-segmentation. We believe that probabilitic relaxation could be pursued in the future as a way to smoothen the probability map, and the usual postsegmentation region merging techniques could also be employed.

\section{REFERENCES}

[1] P. S. U. Adiga and B. B. Chaudhuri. An efficient method based on watershed and rule-based merging for segmentation of $3-D$ histo-pathological images. Pattern Recognition, 34(7):1449-1458, 2001.

[2] S. Beucher and C. Lantuéjoul. Use of watersheds in contour detection. In International Workshop on Image Processing, pages 2.1-2.12, Rennes, September 1979. CCETT/IRISA.

[3] S. Beucher and Centre De Morphologie Mathmatique. The watershed transformation applied to image segmentation, June 281991.

[4] J. C. Bezdek. Pattern Recognition with Fuzzy Objective Function Algorithms. Plenum Press, New York, 1981.

[5] C. Ortiz de Solorzano, E. Garcia Rodriguez, and et al A. Jones. Segmentation of confocal microscope images of cell nuclei in thick tissue sections. Journal of Microscopy, 193(3):212-226, 1999.

[6] R. Deriche. Using canny's criteria to derive a recursively implemented optimal edge detector. International Journal of Computer Vision, 1(2):167-187, 1987.

[7] Sébastien Derivaux, Sébastien Lefèvre, Cédric Wemmert, and Jerzy Korczak. Watershed segmentation of remotely sensed images based on a supervised fuzzy pixel classification. 2006.

[8] M. Frucci. A novel merging method in watershed segmentation. In ICVGIP, 2004.

[9] C. Lantuéjoul. La squelettisation et son application aux mesures topologiques des mosaïques polycristallines. $\mathrm{PhD}$ thesis, Ecole des Mines de Paris, 1978.

[10] O. Lezoray. Segmentation d'images par morphologie mathématique et classification de données par réseaux de neurones: Application à la classification de cellules en cytologie des séreuses. Ph.d thesis, University of Caen, 2000.

[11] G. Lin, U. Adiga, K. Olson, J. F. Guzowski, C. A. Barnes, and B. Roysam. A hybrid 3D watershed algorithm incorporating gradient cues and object models for automatic segmentation of nuclei in confocal image stacks. Cytometry Part A, 56A(1):23-36, 2003.

[12] N. Malpica, C. Ortiz, J.J. Vaquero, A. Santos, I. Vallcorba, J.M. García-Sagredo, and F. del Pozo. Applying watershed algorithms to the segmentation of clustered nuclei. Cytometry, 28(4):289-297, 1997.

[13] D. R. Martin. An empirical approach to grouping and segmentation. In Ph.D., 2002.

[14] F. Meyer. Topographic distance and watershed lines. Signal Processing, 38(1):113-125, July 1994.

[15] F. G. Meyer and S. Beucher. Morphological segmentation. Journal of Visual Communication and Image Representation, 1(1):21-46, 1990.

[16] L. Najman and M. Schmitt. Watershed of a continuous function. Signal Processing, 38:99-112, 1994.

[17] N. Otsu. A threshold selection method from gray-level histograms. IEEE Transactions on Systems, Man, and Cybernetics, 9(1):62-66, 1979.

[18] S. Philipp-Foliguet and L. Guigues. Multi-scale criteria for the evaluation of image segmentation algorithms. Journal of Multimedia, 3(5), 2008.

[19] A. Rosenfeld and J. Pfaltz. Distance functions on digital pictures. Pattern Recognition, 1:33-61, 1968. 
[20] V. Vigneron, T. Q. Syed, G. Barlovatz-Meimon, M. Malo, C. Montagne, and S. Lelandais. Adaptive filtering and hypothesis testing: Application to cancerous cells detection. Pattern Recognition Letters, 31(14):2214-2224, 2010.

[21] L. Vincet. Segmentation et Mise en Correspondance de $R$ 'egions de Paires d'Images Stéréoscopiques. $\mathrm{PhD}$ thesis, Université Paris IX - Dauphine, July 1991.

[22] C. Wählby, I.-M. Sintorn, F. Erlandsson, G. Borgefors, , and E. Bengtsson. Combining intensity, edge, and shape information for $2 d$ and $3 d$ segmentation of cell nuclei in tissue sections. Journal of Microscopy, 215(1):67-76, 2004.

[23] C. Wählby, I.-M. Sintorn, and et al F. Erlandsson. Combining intensity, edge, and shape information for $2 d$ and $3 d$ segmentation of cell nuclei on tissue sections. Journal of Microscopy, 215(1):67-76, July 2004.

[24] D. L. Wilson, A. J. Baddeley, and R. A. Owens. A new metric for grey-scale image comparison. International Journal of Computer Vision, 24(1), 1997.

[25] W. A. Yasnoff, W. Galbraith, and J. W. Bacus. Error measures for objective assessment of scene segmentation algorithms. AQC, 1:107-121, 1979.

[26] Y. J. Zhang. Evaluation and comparison of different segmentation algorithms. Pattern Recognition Letters, 18:963-974, 1997. 